\section{Yoga for persons with severe visual impairment: a feasibility study}

\author{
Pamela E. Jeter, 1 Gislin Dagnelie, 1 \\ Sat Bir S. Khalsa,2 Steffany Haaz, 3 \\ Ava K. Bittner1 \\ 1Wilmer Eye Institute, Johns Hopkins \\ University; 2Brigham and Women's \\ Hospital, Harvard Medical School; \\ ${ }^{3}$ Corporate Health Solutions, \\ Baltimore, USA
}

\section{Abstract}

This exploratory study aims to establish the feasibility of an Ashtanga-based Yoga Therapy (AYT) program for improving sleep disturbances, balance, and negative psychosocial states, which are prevalent issues for visually impaired (VI) individuals. Ten legally blind adult participants were randomized to an 8 -week AYT program. Four subjects in the 1 st cohort and three in the $2^{\text {nd }}$ cohort successfully completed the AYT program. They convened for one session per week with an instructor and performed two home-based sessions per week using an audio CD. The Pittsburgh Sleep Quality Index (PSQI), Perceived Stress Scale (PSS), Beck Anxiety Inventory (BAI), and Beck Depression Inventory (BDI) were administered at baseline and post-intervention. A Timed 0ne-Leg balance measure, respiratory rate (RR), and the Philadelphia Mindfulness Scale (PHLMS) were assessed in the $2^{\text {nd }}$ cohort. Both groups completed a qualitative exit survey. Positive exit survey responses (all subjects were extremely or mostly satisfied, and wanted to continue AYT) and good participation rates (7 subjects attended at least 7 of the 8 weekly sessions) support the feasibility of the AYT. PSQI, PSS, BAI and BDI scores changed in the direction of reduced negative symptoms after AYT for the $1^{\text {st }}$ cohort. Changes in PSOI and PSS for the 2nd cohort were varied. Balance, RR and PHLMS awareness trended toward improvement for each individual. This preliminary study provides proof of concept for potential benefits of AYT that may be observed in VI subjects. Larger studies and an active control group are needed to determine efficacy.

\section{Introduction}

Visual impairment (VI) is considered among the 10 most prevalent causes of disability in the United States and approximately 1 in 28 Americans older than 40 years are legally blind. ${ }^{1}$ Most current research for VI is focused on the development of treatments such as pharmacologic agents, gene therapy, stem cells and prosthetic devices. ${ }^{2,3}$ However, not all patients benefit from these proposed treatments, and research is also needed to understand and improve patients' disease-related symptoms that often result in reduced quality of life. ${ }^{4}$

Alternative therapies such as yoga have gained popularity in the U.S. in the past decade ${ }^{5,6}$ and have been reported to increase well-being and quality of life for the normally sighted population.7,8 In an on-line survey, physical and emotional well-being were reported as motivating factors for individuals with retinitis pigmentosa (RP) (a slowly progressive retinal degenerative disease) seeking alternative therapies such as yoga and meditation. Of the patients surveyed, $31 \%$ tried yoga and of those, $93 \%$ reported improved stress, fatigue and anxiety levels. ${ }^{9}$ Despite growing interest, yoga as a treatment for these symptoms has not been systematically studied in the VI population.

Reductions in sleep quality in persons with retinal degenerative disease and advanced

vision loss may be related to decreased light processing due to reduced functioning of retinal photosensitive cells and narrowing of the field of view.10,11 Sleep disturbances in RP, characterized by reduced alertness, disturbed nighttime sleep, and greater daytime sleepiness, were found to be greater than in agematched normally sighted individuals, and may be due in part to photoreceptor loss. ${ }^{12}$ Photosensitive retinal ganglion cells containing the photopigment melanopsin have been identified as essential to circadian photoentrainment. 13

Recently, Altimus et al. further demonstrated that rod and cone photoreceptors, in addition to ganglion cells containing melanopsin, help modulate the light-dark cycle in mice. ${ }^{14}$ Other studies have reported a relationship between RP and abnormal melatonin production affecting the circadian cycle in some patients.10,11 Taken together, this raises the possibility that as vision loss progresses, reductions in retinally mediated light signals affect the release of melatonin leading to the observed sleep disturbances. Studies with normally sighted individuals practicing yoga have revealed positive results on subjective sleep measures ${ }^{15-17}$ and increased physiological measures of melatonin, ${ }^{18-20}$ although these studies are potentially confounded by the lack of control for nighttime light exposure. Melatonin contributes to sleep onset and sleep onset latency or SOL, a measure ${ }^{21}$ that can be reliably detected by sleep questionnaires, ${ }^{16}$ is considered an indicator of the duration it takes to fall asleep. Persons with ocular disease that have difficulty initiating sleep may be subject
Correspondence: Pamela E. Jeter, Lions Vision Center, Wilmer Eye Institute $550 \mathrm{~N}$. Broadway, 6th Floor, Baltimore, MD 21205, USA

Tel. +1.410.5026434 - Fax: +1.410.955.1829

E-mail: pjeter1@jhmi.edu

Contributions: PEJ, performed experiments, conceived the AYT protocol, analyzed data and wrote the paper; AKB, GD, supervised the study. All authors discussed the design, results and implications and commented on the manuscript at all stages.

Key words: yoga, sleep, stress, mindfulness, balance.

Acknowledgements: we would like to thank Joe Ingber, RYT, for volunteering his time as instructor for the yoga program. We would also like to thank the National Federation of the Blind and St. George's Church for providing additional space for the yoga classes. This exploratory study was funded by The Mona Wong Chou Wilmer Research Pilot Grant and the Johns Hopkins School of Public Health Delta Omega Scholarship.

Conflict of interests: the authors report no conflict of interests.

Received for publication: 30 September 2011 Revision received: 27 February 2012.

Accepted for publication: 28 February 2012.

This work is licensed under a Creative Commons Attribution NonCommercial 3.0 License (CC BYNC 3.0).

(C) Copyright P.E. Jeter et al., 2012

Licensee PAGEPress, Italy

Alternative Medicine Studies 2012; 2:e5

doi:10.4081/ams.2012.e5

to sleep disturbances. A subjective global measure of sleep disturbance and SOL was used in this study to obtain preliminary information regarding sleep problems in this population. Obtaining measures of melatonin were beyond the scope of this project.

The impact of vision loss on negative mood states (e.g. anxiety, depression, stress) may be an independent factor contributing to disturbed sleep. Transient episodes of reduced vision have been correlated with increases in perceived stress and negative mood in RP.22,23

Functional loss of vision is a risk factor for depression and anxiety in the aging VI population. ${ }^{24}$ In general, negative psychosocial states that accompany loss of vision are often unrecognized and remain untreated. 4 Positive results after yoga have been reported in the general population for stress, ${ }^{25}$ depression ${ }^{26}$ and anxiety. ${ }^{27}$ It has also been suggested that simply modifying patterns of improper breathing can lead to significant improvements in psychological symptoms. ${ }^{28}$ Yogic breathing is a 
technique yielding benefits for psychological distress such as stress, anxiety, depression as well as sleep. ${ }^{29}$ Respiratory rate (RR) has been associated with cardiovascular health and stress $^{30}$ and RR may be an indicator of improved stress and related factors. 31 Thus, implementing a yoga regimen with a breath component may provide a long-lasting foundation for breathing that could have an added beneficial effect to reduce stress and anxiety.

Furthermore, vision plays a dominant role in a sighted person's ability to navigate through the environment. Accordingly, the loss of vision, accompanied by a decrease in balance, postural stability, and mobility, may increase injury and reduce independence and quality of life in VI patients. ${ }^{32-37}$ As vision declines, adopting training strategies that enhance the use of other sensory information may aid in the development of awareness of the body as it moves in space. In an eyes-closed condition, a group trained in yoga was able to retain balance on a vertical force platform better than a control group trained in physical exercise, indicating they were better able to use proprioceptive cues. ${ }^{38}$ The ability to stand on a single-leg is an important predictor of falls in the elderly. ${ }^{39}$ Due to the anticipated increase in the next 20 years of the legally blind aging population, elderly individuals in late-stages of acquired vision loss (e.g. age-related macular degeneration; RP) represent an important demographic to consider. ${ }^{1}$ Balance is a critical prerequisite to movement 40 and has been shown to be sensitive to change over time. ${ }^{41}$ Balance improved substantially in a separate study using a timed, one-legged balance test after yoga training in healthy adults ${ }^{42}$ and in patients with osteoporosis. ${ }^{43}$ Therefore, cultivating the integration of mind and body through yoga may yield favorable outcomes as a means to improve balance.

Ashtanga is a system of yoga taught by Sri K. Pattabi Jois in Mysore, India. It is an integrated system of asanas (postures), vinyasa (movement), and ujjayi (victorious breath) and is easily implemented regardless of age or level of experience. The main tenet of Ashtanga is movement with the breath. Ujjayi breathing is a specialized technique that emphasizes use of the diaphragm. Observing the quality of breath can be a diagnostic tool for the quality of the practice. In this sense, practicing asana becomes the vehicle for teaching proper breathing techniques. Most importantly, the student is taught to find a balance between what is achievable in an asana while providing room for growth to move deeper into a posture simply by listening to their ujjayi breathing. This makes Ashtanga a suitable, gentle practice since it does not emphasize flexibility or strength as a goal. Instead, the goal is to synchronize postures with the breath while keeping a steady, rhythmic breathing pattern. Strength and flexibility are by-products after persistent practice. The Ashtanga-based Yoga Therapy (AYT) is a highly modified Ashtanga yoga sequence developed specifically for the visually impaired population by the author (PEJ). This style of yoga may be beneficial for this population because it combines all elements of a mindfulness practice through breath, postures and movement. With continued practice, the sequence becomes a moving meditation. Mindfulness in this setting promotes self-awareness in a noncompetitive, non-judgmental environment and at the same time promotes awareness of muscular movements, alignment, mental states and breath. 44

Increased levels of mindfulness were found after yoga in a healthy population 45 and a chronic illness population. ${ }^{17}$ A mindfulness assessment was included in this study to evaluate the potential to develop increased mindfulness in the AYT.46 By pursuing an integrated approach, this preliminary research study aimed to determine the safety and efficacy of an AYT geared towards the VI population and whether it could have an immediate and com- prehensive impact on secondary symptoms and quality of life for individuals with severe vision loss. The primary goals of this proof of concept study were to evaluate safety and feasibility of the AYT for VI to: i) reduce sleep disturbances, ii) improve psychosocial indicators, and iii) improve balance and RR.

These data are especially important since to our knowledge there have been no previous publications involving yoga interventions for attendant symptoms experienced by the $\mathrm{VI}$ population.

\section{Materials and Methods}

\section{Participants}

Table 1 lists the participants' demographics, including age, gender, group assignment, ocular disease diagnosis, and level of vision. Ten participants (mean age of $46 \pm 12$ years) were recruited from the Low Vision Clinic of the Wilmer Eye Institute at Johns Hopkins Hospital and from local community-based listings (e.g. National Federation of the Blind, Northern Virginia American Council for the Blind) through email and flyers to prospective participants.

Participants were limited to a small sample of 10 set a priori for the purposes of establishing feasibility and safety before a larger trial is set in motion. Participants were included in the study if: (a) they were older than 18 years of age (b) they were diagnosed with legal blindness on the basis of visual field diameter $<20^{\circ}$ as determined by Goldmann and/or Humphrey visual field, 47 or on the basis of best-corrected visual acuity (VA) $<20 / 200$ as determined with the Early Treatment of Diabetic Retinopathy Study (ETDRS) chart, 48 (c) the ocular disease stage was expected to remain relatively stable throughout a 3-6 month period, and (d) the participant was

Table 1. Participant demographics.

\begin{tabular}{|c|c|c|c|c|c|c|c|}
\hline SBJ ID & Age & Gender & Cohort & Disease & ETDRS VA & P-R CS $(\log C S)$ & GVF \\
\hline SBJ01 & 56 & M & 1 st & $\mathrm{RP}$ & BLP & BLP & BLP \\
\hline SBJ02 & 46 & $\mathrm{~F}$ & 1 st & Nystagmus, Congenital Defects & BLP & BLP & BLP \\
\hline SBJ03 & 59 & $\mathrm{~F}$ & 1 st & Acute Retinal Necrosis & $20 / 180$ & $\mathrm{n} / \mathrm{a}$ & $<20^{\circ} *$ \\
\hline SBJ04 & 31 & $\mathrm{~F}$ & 1 st & $\mathrm{RP}$ & $20 / 50$ & 1.95 & $<20^{\circ} * *$ \\
\hline SBJ05 & 56 & $\mathrm{~F}$ & 1 st & Retinopathy of Prematurity & $20 / 800$ & $\mathrm{n} / \mathrm{a}$ & $\mathrm{n} / \mathrm{a}$ \\
\hline SBJ06 & 50 & M & $2^{\text {nd }}$ & $\mathrm{RP}$ & $20 / 126$ & 0.10 & $<20^{\circ} *$ \\
\hline SBJ07 & 30 & $\mathrm{~F}$ & $2^{\text {st }}$ & Nystagmus, Congenital Cataracts, Photophobia & $20 / 400$ & $\mathrm{n} / \mathrm{a}$ & n/a \\
\hline SBJ08 & 30 & $\mathrm{~F}$ & $2^{\mathrm{st}}$ & $\mathrm{RP}$ & $20 / 20$ & 1.60 & $<20^{\circ} * *$ \\
\hline SBJ09 & 47 & M & $2^{\text {st }}$ & $\mathrm{RP}$ & $20 / 152$ & 0.55 & $<20^{\circ} * *$ \\
\hline SBJ10 & 58 & $\mathrm{~F}$ & 2 st & $\mathrm{RP}$ & BLP & BLP & BLP \\
\hline
\end{tabular}

P-R CS, Pelli-Robson Contrast Sensitivity; ETDRS VA, Early Treatment Diabetic Retinopathy Study Visual Acuity; GVF, Goldmann Visual Field; n/a, vision too poor to obtain reliable results; BLP, Bare Light Perception; RP, Retinitis Pigmentosa. Legal blindness $=\mathrm{GVF}<20^{\circ}$ or best-corrected VA $<20 / 200$ on the ETDRS; normal CS is $>1.80 \log$ CS in young adults and $>1.65$ logCS in older adults. *V4e; **III4e. 
healthy to the extent that participation in a yoga program would not exacerbate any existing disease conditions. Participants were excluded on the basis of (a) clinically diagnosed or significant sleep disorder (e.g., sleep apnea) or a medical condition (e.g., chronic pain) responsible for sleep complaints, (b) use of prescription sleep medication more than once a week for duration of the study, (c) use of other psychotropic medication, or (d) consumption of $>2-3$ alcoholic beverages per day or smoking $>10$ cigarettes per day. The protocol for the study was approved by the Institutional Review Board of the Johns Hopkins University School of Medicine and followed the tenets of the Declaration of Helsinki. The consent form was read to participants due to their visual impairment. All participants provided informed consent.

\section{Study design}

This proof of concept study determined the feasibility of the AYT in two separate cohorts of VI subjects. Participants were randomized to the first or second 8-week AYT. The $2^{\text {nd }}$ cohort was invited to participate in the AYT after the first group's completion of the AYT. Two cohorts allowed us to manage teacher-student interactions in smaller class sizes. It also minimized the burden to the participants' regarding questionnaires and transportation and allowed us to pilot additional measures with the second group based on experience with the first group.
Comprehensive vision tests were conducted at baseline that included VA, Pelli-Robson contrast sensitivity (P-R CS) ${ }^{49}$ and Goldmann visual fields (GVF) ${ }^{47}$ for both groups. Psychosocial and sleep questionnaires were administered at two time points for both groups: once at baseline before the first 8week yoga intervention and once immediately after the intervention period.

When possible, the questionnaires were administered online via SurveyMonkey.com, LLC (Palo Alto, CA) or by email on a word document, and participants used their remaining vision and/or accessibility software to selfadminister the questionnaires. In situations where the participant did not have access to a computer or support from a caregiver $(n=2)$, the questionnaires were read aloud and responses recorded by the researchers via phone. After initial feasibility was noted with the first AYT group, we piloted three additional measures during the 2 nd cohort's participation in the AYT: a mindfulness questionnaire administered at baseline, week four and postintervention, and a balance measure and respiratory rate (RR) measured before and after the AYT. A brief exit survey was collected immediately following both groups' participation in the yoga program. Participants received a free yoga mat and $\$ 25$ for their participation in the study. All procedures took place at one of three locations selected to provide accessibility for our visually impaired population, i) Lions Vision Center (LVC, Wilmer Eye Institute, Baltimore, MD), ii) National Federation of the
Blind (NFB, downtown Baltimore, MD) and iii) St. George's Episcopal Church (Arlington, VA).

\section{Intervention}

Participants took part in an orientation session before the yoga program began, in order to familiarize them with the style of yoga, the ujjayi breathing technique, and alignment techniques using the mat, class etiquette, and modifications. Participants in each group convened once per week for eight weekly one-hour sessions with the author (PEJ). Participants were provided with an audio CD developed by the author to practice at home and were encouraged to practice at least twice a week (i.e. equivalent to approximately 16 home practice sessions during the intervention period). The Ashtanga-based Yoga Program (AYT) is a highly modified Ashtanga yoga sequence developed specifically for the visually impaired population by the author (PEJ), a trained Ashtanga teacher of four years and practitioner of 7 years, on the basis of her experience teaching blind students at the Braille Institute in Los Angeles. Teaching yoga to those with VI requires simple modifications to postures, clear descriptions, and hands-on adjustments. Each class began with simple seated breathing, a warm-up, standing postures, seated postures, followed by breathing and a final resting pose. Table 2 lists the full sequence of poses taught during AYT. Each pose was held for five breaths or for as long as the subject was able. Each class included a question and answer

Table 2. Details of Ashtanga-based yoga therapy.

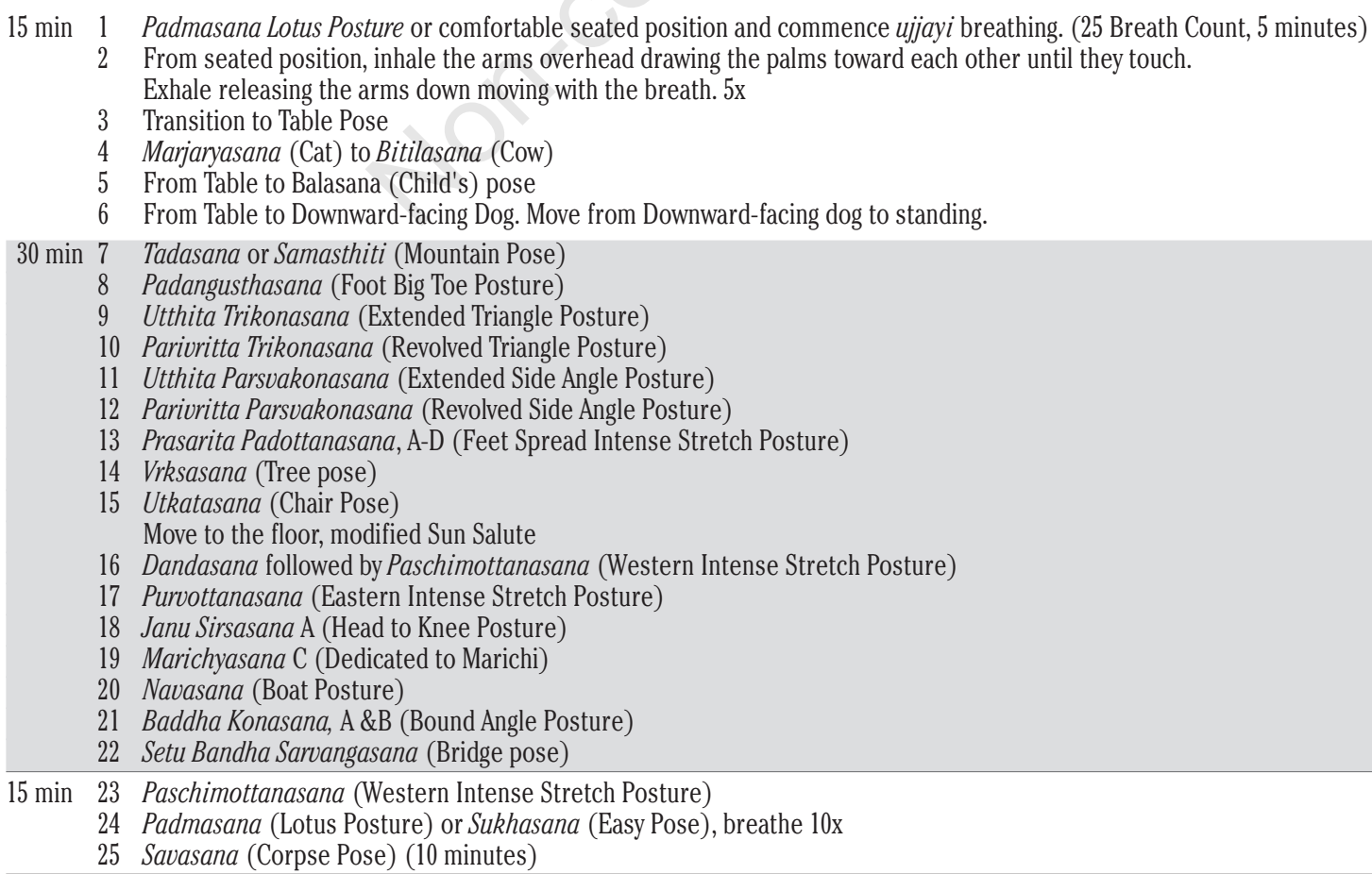


period at the beginning and end of class.

An additional instructor participated during the $2^{\text {nd }}$ cohort's AYT participation. The second instructor had experience teaching yoga to students with physical disabilities (multiple sclerosis) and was trained to deliver AYT by the author (PEJ) who familiarized him with the protocol and needs of the VI population. The author (PEJ) observed 5 of the 8 classes taught by the second instructor to help ensure fidelity. The AYT is amenable to study because it is composed of a standardized sequence of postures held for a fixed duration. While the sequence of asanas remain the same each session, each asana is modified to suit the individual's needs. Study patients are more likely to comply with an intervention if it is safe, engaging and easy to follow. 50

\section{Study outcomes}

The 19-item Pittsburgh Sleep Quality Index (PSQI) questionnaire was used to gauge sleep quality over the past month (Cronbach alpha estimate $=0.83) .{ }^{51}$ It includes both qualitative and quantitative aspects of sleep, and evaluates seven subscale dimensions of sleep quality. Of interest in this study were the global score and sleep onset latency (SOL) sub scale score. We administered three brief online questionnaires to assess various psychosocial states: the Beck Anxiety Inventory (BAI), 52 Beck Depression Index (BDI) ${ }^{53}$ and the Perceived Stress Scale (PSS). ${ }^{54}$ The 21-item BAI was used to measure the severity of an individual's anxiety over the past month (Cronbach alpha estimate ranges from 0.92 to 0.94).52 The 21-item BDI provided a measure of severity of depression (Cronbach alpha estimate $=0.81) .55$

The 14 item PSS subjectively assessed the degree to which respondents appraised situations in their life to be stressful on a given day (Cronbach alpha estimate $=0.85) .54$ The questionnaires have been previously validated in other populations, are reliable and routinely used for clinical and research purposes. 17,25,26,56-59 The questionnaires were completed online through an internet website or over the phone, depending on the subject's ability to access the computer.

The 2nd cohort completed only the PSQI and PSS plus 3 additional new measures. The PSS for the $2^{\text {nd }}$ cohort was administered on a weekly basis to assess potential fluctuations in appraised stress. We did not include the BDI and BAI in order to reduce the burden on the $2^{\text {nd }}$ cohort participants since additional measures were added (PHLMS, respiratory rate, balance).

Changes in balance were assessed for individuals in the $2^{\text {nd }}$ cohort during their participation in the AYT. The Timed One-Leg Stance protocol requires the subject to raise one foot off the ground with their arms down at their sides, and the time up to 30 seconds is recorded until any touch down of a foot or hand to a surface occurs. The task is performed 3 times and the best of 3 is chosen. ${ }^{60-62}$ Respiratory rate (RR) was measured three times for each participant to reduce within-session variability during baseline and immediately following the 8-week AYT. It was determined by counting the number of inhalations with a stethoscope for $30 \mathrm{sec}$ onds at rest and multiplying by two. 63 The normal adult rate is 12 to 18 breaths/minute. ${ }^{64-66}$

The Philadelphia Mindfulness Scale (PHLMS) is a 20-item scale to assess two independent components of mindfulness: acceptance and awareness. 67 Total scores on both subscales range from 20 to 100 , higher scores reflect greater mindfulness. The PHLMS was collected at baseline, during week 4 and postwaitlist participation. The PHLMS shows good internal reliability as reported in clinical and non-clinical samples (Awareness Cronbach alpha estimate $=0.85 \&$ Acceptance Cronbach alpha estimate $=0.87) .67$ The PHLMS was used as a means to appraise the AYT as a practice that develops awareness and mindfulness in general. It has the advantage of two independent subscales that allow us to detect critical differences in two components of mindfulness. ${ }^{67,68}$ We measured adherence with practice logs and participant experience through exit surveys to further help evaluate the yoga intervention. Participants completed a weekly $\log$ online to report home practice and were used to evaluate adherence. All participants completed a brief exit survey regarding their experience with the AYT. The survey was comprised of open-ended and multiple-choice response items.

The small sample size in this proof of concept study was not powered for statistical comparisons of changes in the questionnaire scores, ${ }^{69}$ and therefore, ANCOVA analyses of our results were not statistically significant. We were limited to evaluating the data based on descriptive statistics and no rigorous statistical analysis of the outcomes is presented here. Instead we focus on the direction of change or trends on an individual basis for each measure (Tables 3-5).

\section{Results}

\section{Study participation and baseline characteristics}

Ten legally blind participants (Table 1) who met our study criteria were enrolled in the study. Four of the five participants in the 1st cohort attended at least 7 of the 8 weekly classes, which was the attendance compliance criteria defined a priori. One participant in the first group missed two classes during the 8week intervention, reported that she failed to follow the home practice instructions, and began taking sleeping pills, which were study exclusion criteria. Therefore, only sleep and psychosocial data for four subjects in the 1st cohort are presented in Table 3 . We were unable to remove the non-compliant subject's responses from the exit survey since it was anonymous. Only 3 participants in the $2^{\text {nd }}$ cohort completed the 8 -week yoga program by attending 7 of the 8 weekly scheduled classes. Two participants did not participate due to

Table 3. Outcome measures completed pre-post- in the Ashtanga-based yoga therapy.

\begin{tabular}{|c|c|c|c|c|c|c|c|c|c|c|c|c|c|c|c|}
\hline \multirow[b]{2}{*}{ SBJ ID } & \multicolumn{3}{|c|}{ PSQI -global } & \multicolumn{3}{|c|}{ PSQI-SOL (mins) } & \multicolumn{3}{|c|}{ PSS } & \multicolumn{3}{|c|}{ BAI } & \multicolumn{3}{|c|}{ BDI } \\
\hline & pre & post & trend & pre & post $t$ & trend & pre & post & trend & pre & post & trend & pre & post & trend \\
\hline SBJ01 & 2 & 1 & - & 15 & 10 & - & 11 & 7 & - & 7 & 2 & - & 8 & 8 & $\mathrm{x}$ \\
\hline SBJ02 & 5 & 4 & - & 15 & 15 & $\mathrm{x}$ & 11 & 4 & - & 6 & 0 & - & 2 & 0 & - \\
\hline SBJ03 & 15 & 8 & - & 120 & 60 & - & 21 & 20 & - & 6 & 4 & - & 11 & 5 & - \\
\hline SBJ04 & 10 & 4 & - & 60 & 30 & - & 28 & 14 & - & 14 & 2 & - & 4 & 3 & - \\
\hline SBJ06 & 12 & 11 & - & 60 & 150 & + & & & & & & & & & \\
\hline SBJ07 & 4 & 5 & + & 10 & 5 & - & & & & & & & & & \\
\hline SBJ010 & 3 & 5 & + & 60 & 30 & - & & & & & & & & & \\
\hline Mean & $7.3(5.0)$ & $5.4(3.2)$ & - & $48.6(39.2)$ & $42.9(50.7)$ & - & $17.8(8.3)$ & $11.3(7.2)$ & - & $8.3(3.9)$ & $2.0(1.6)$ & - & $6.3(4.0)$ & 4.0 (3.4)- & \\
\hline
\end{tabular}

First cohort SBJ0-04, second cohort SBJ06,07,10. PSQI, Pittsburgh Sleep Quality Index; PSQI-SOL, Pittsburgh Sleep Quality Index-Sleep Onset Latency; PSS, Perceived Stress Scale; BAI, Beck Anxiety Inventory; BDI, Beck Depression Inventory. Trend: post scores minus baseline; higher score is worse; - indicates trend for reduction of symptoms (improvement); + indicates worsening of symptoms; $x$ indicates no change. 
scheduling conflicts. The $2^{\text {nd }}$ cohort did not begin their participation until after the initial cohort completed the AYT ( $\sim$ month), which may have introduced new, unpredictable scheduling conflicts not present during the initial recruitment period. At least one subject opted not to participate in the AYT since her schedule became too busy. Another $2^{\text {nd }}$ cohort participant dropped out after 4 sessions of the AYT because he became unable to attend regularly scheduled classes due to his conflicting responsibilities related to family caregiving. This participant also failed to return the home practice log.

Overall weekly class attendance was 7.5 (range 7-8; SD 0.6) and 7 (SD 0) while individual home practice compliance was 14 (range $10-16$; SD 2.8) and 14 (range 13-16; SD 1.7) as reported in weekly practice logs, across the 4 participants in the first AYT group and 3 participants in the $2^{\text {nd }}$ cohort group who completed the intervention, respectively. The baseline characteristics were evenly balanced between cohorts and not statistically significantly different between the two groups. No serious adverse or non-serious events were reported.

\section{Outcome measures}

Individual results for the questionnaires completed pre- and post- AYT and are presented in Table 3, and improvements in the mean scores were noted across all four questionnaires. No associations were found for severity of vision loss and the outcome measures. We suspect this may be due to the limited range of vision in our limited sample. The PSQI global, PSQI_SOL, PSS, BDI and BAI scores for the first cohort group (SBJ01-04) all changed in the direction of reduced negative symptoms after the yoga intervention, with the exception of two instances that showed no change (Table 3). The PSQI SOL scores for the first AYT group mostly changed in the direction of reduced negative symptoms ( $\mathrm{n}=3$ of 4 changed by approximately $45 \%$ or $31.7 \mathrm{~min}$ ) and one showed no change after the yoga intervention. In addition, three subjects (SBJ03, SBJ04 and SBJ06) met the criteria for sleep disturbances according to their PSQI-Global score $(>5)$ at baseline and showed improvements in their scores post-AYT. Three of four participants in the 1 st cohort showed reduced BDI scores and one showed no change. The greatest amount of change in the BDI was reported by the subject (SBJ03) who had greater than minimal depressive symptoms at baseline (BDI $\geq 10$ ). BAI scores improved across all participants in the first cohort, and the largest changes on average were noted for the BAI.

Sleep and PSS data are presented in Tables 3 (bottom) and 4 for the 2nd cohort (SBJ06, SBJ07, SBJ10). Changes in the PSQI-global scores were marginal and mixed. It's interesting to note that the direction of change in the global score was exactly the opposite for the SOL score directions, something we have no explanation for. The PSS was administered weekly for the $2^{\text {nd }}$ cohort in order to capture the degree of variability that might be observed due to acute stress (vs. chronic). Indeed, the scores in Table 4 are somewhat volatile during the course of the program. Merely looking at pre- and post- scores show one subject getting worse, the other getting better and the last one showing no change. In all cases, there is evidence of better and worse scores throughout the intervention for each subject suggesting that perhaps the PSS is not the best measure to capture treatment effects. This also highlights the inherent variability within individual subjects. However, without a much larger sample size, it is difficult to draw any substantive conclusions from these measures in this study. During the $2^{\text {nd }}$ cohort group's participation in the AYT, three additional measures were piloted for feasibility (Table 5). Both RR and balance measures improved after the intervention for all three individuals (Table 5). The PHLMS showed a positive trend for improved awareness subscale scores per individual but not for the acceptance subscale.

\section{Exit survey results}

All subjects who completed the AYT $(n=8)$ completed an anonymous exit survey after the AYT. All subjects reported being extremely $(5 / 8)$ or mostly satisfied (3/8) with the yoga research program. Most participants reported that they thought the AYT helped with stress (5/8) and balance (5/8). Some (3/8) subjects reported that yoga helped with sleep. Almost all (7/8) subjects reported that yoga helped with relaxation and focus through yoga breathing techniques. All participants (8/8) expressed an interest in participating in a yoga program like this in the future in order to learn more yoga skills, lower stress, practice breathing skills, stretching and balance, and moreover would recommend the AYT to a friend (8/8) in order to receive the same benefits.

All participants reported being extremely (5/8) or mostly honest (3/8) when reporting their daily practice in their practice logs. All participants (8/8) reported really liking the yoga instructors and really or mostly liking the setting and environment of the yoga program (5/8 Really liked and 3/8 Mostly liked). Most liked the audio CD (7/8) for home practice. Two subjects expressed a preference for having separate CDs for the early parts of the study and for later after becoming more familiar with the sequence. When asked to describe their experience with the yoga practice at home, half found practicing at home easier as the program progressed (4/8); however, motivation and commitment were identified as factors that prevented regular home practice $(3 / 7)$.

Table 4. Perceived Stress Scale for 2nd Cohort collected on a weekly basis.

\begin{tabular}{lcccccccc} 
SBJ ID & Baseline & Week 2 & Week 3 & Week 4 & Week 5 & Week 6 & Week 7 & Week 8 \\
SBJ06 & 11 & 12 & 5 & 7 & 13 & 0 & 9 & 14 \\
SBJ07 & 18 & 22 & 16 & 16 & 27 & 11 & 9 \\
\hline SBJ10 & 14 & 10 & 13 & 7 & 13 & 14 & 17
\end{tabular}

Table 5. Outcome measures at baseline and post-intervention for second group.

\begin{tabular}{|c|c|c|c|c|c|c|c|c|c|c|c|c|c|c|}
\hline \multirow[b]{2}{*}{ SBJ ID } & \multicolumn{4}{|c|}{ PHLMS-Acceptance } & \multicolumn{4}{|c|}{ PHLMS-Awareness } & \multicolumn{3}{|c|}{ Balance (sec) } & \multicolumn{3}{|c|}{ Respiratory rate } \\
\hline & pre & Week 4 & post & trend & pre & Week 4 & Post & trend & pre & post & trend & pre & post & trend \\
\hline SBJ06 & 37 & 35 & 42 & - & 38 & 38 & 41 & - & 3.55 & 5.31 & - & 16 & 12 & - \\
\hline SBJ07 & 36 & 34 & 31 & + & 35 & 35 & 39 & - & 23.52 & 30 & - & 18 & 12 & - \\
\hline SBJ010 & 33 & 29 & 27 & + & 47 & 48 & 49 & - & 3 & 3.7 & - & 20 & 12 & - \\
\hline Mean & $35.3(2.1)$ & $32.7(3.2)$ & $33.3(7.8)$ & + & $40(6.3)$ & $40.3(6.8)$ & $43.0(5.3)$ & - & $10(11.7)$ & $13(14.7)$ & - & $18(2.0)$ & 12 & - \\
\hline
\end{tabular}

PHLMS; Philadelphia Mindfulness Scale. Trend: post scores minus baseline; higher score is worse; - indicates trend for reduction of symptoms (improvement); + indicates worsening of symptoms. 


\section{Discussion}

In this preliminary study, the feasibility and initial safety of the AYT were demonstrated in a small group of individuals with VI. The positive exit survey responses and relatively good participation rates support the feasibility of the AYT. All participants who completed the study reported the desire to continue with the AYT and found it enjoyable. In general, the AYT appeared to provide overall benefits for sleep and psychosocial factors for the first group of participants. Improvements in sleep and PSS were not consistent across the members of the $2^{\text {nd }}$ cohort. RR and balance were only assessed in the $2^{\text {nd }}$ cohort participants, but all three subjects showed improvements in both of these measures after their AYT participation. This preliminary study provides proof of concept for potential benefits that may be expected from the AYT in a population with VI.

The mean reduction in PSS scores observed in the first cohort of 4 participants in our study (36.5\%) was greater than another study that found a $29.5 \%$ decrease in scores after a stress reduction program. ${ }^{70}$ The PSS may vary significantly from week-to-week in some people, potentially leading to chance reductions or regression toward the mean in the control group when only comparing a single pre- and post-intervention measure. Furthermore, the PSS timeframe assessed stress in the past week, which may have identified acute episodes rather than capture a global measure of chronic stress in the 1 st cohort. As evidenced in the $2^{\text {nd }}$ cohort, fluctuations were observed from week to week rendering the results difficult to interpret.

Planning assessments with a longer timeframe (e.g. 2 weeks) may provide a more accurate assessment of perceived stress. ${ }^{54}$

The improvements in the BAI across the four subjects in the $1^{\text {st }}$ cohort were quite large compared to a yoga study by Harner et al. with a much larger sample size 58 (75.9\% versus $38.9 \%$ ). Compared to two separate studies measuring PSQI-SOL in much larger samples, ${ }^{71,72}$ sleep onset latency in our study changed to a greater degree for the four participants in the $1^{\text {st }}$ cohort ( 32 min vs. $15-18$ min). Only one of the four subjects (SBJ03) in the first AYT group had greater than minimal depressive symptoms (BDI score $\geq 10$ ) at baseline, and therefore, floor effects may have prevented us from observing larger changes. SBJ03's score was reduced by 6 points (55\% reduction) post-intervention comparable to Woolery et al.73 who observed significant reductions in BDI ( average $69.5 \%$ ) after a 5 week yoga intervention for mildly depressed young adults.

The improvements in the RR and timed oneleg stance measures are important because they suggest that the AYT may slow breathing and improve balance in those with VI. Balance is more impaired as vision loss progresses and therefore increases the risk of falls. ${ }^{32,74}$ It has been reported that individuals increase their chances of sustaining an injury due to a fall by two times if they are unable to perform a 0neLegged Stance Test for five seconds. ${ }^{39}$ Two of 3 participants were below five seconds and one improved to greater than 5 seconds after the intervention. The third subject's performance was high to begin with and improved by 6.48 seconds to the maximum attempted test time of 30 seconds. Improvements in balance in our study suggest that participants may be developing strategies that access other sensory cues and increase awareness of where their body is in space. Indeed, the average improvement range observed in our study is comparable to a yoga study for women with postmenopausal osteoporosis who are also at risk for falls. ${ }^{43}$ Future studies of the AYT should assess changes in balance in a sample larger than three VI participants, as well as determine whether changes in balance of this magnitude are clinically significant and may translate into a reduced risk for falls or injuries.

We chose to measure changes in RR in the $2^{\text {nd }}$ cohorts since the main tenet of the AYT is synchronized movement with the breath. Modest improvements were observed in our group; however, a larger sample size is needed in the future to attempt to demonstrate any statistically significant changes.

Focusing on the breath draws the attention inward and at the same time promotes selfawareness to muscular movements and alignment. ${ }^{45}$ Since the AYT contains a component of self-awareness we believed the PHLMS would be a useful measure. We found trends for improved awareness scores in the $2^{\text {nd }}$ cohort group after their participation. It is interesting to note that this difference only emerged towards the end of the program (i.e., scores were not qualitatively different from baseline at week 4 testing), which indicates that cultivating awareness may be a process that improves with practice. The scores for acceptance did not show any substantial changes, and it is possible that acceptance is not a component of the AYT itself or that it takes longer than 8-weeks to manifest. A larger sample size and/or a longer duration program will be needed in the future to critically tease these apart. To our knowledge, this is the first study that uses yoga to reduce secondary symptoms in the VI population. While we view our results as promising, there are some limitations that should be acknowledged. This proof of concept study's limited sample size was not designed or powered to detect statistically significant effects. Also due to the limited sample size, we were unable to detect interactions between measures such as stress and sleep. Self-selec- tion, or other unknown factors may have confounded our results. Scheduling and nonadherence were issues for 3 of the 10 participants. The potential influence of the investigator as yoga instructor may have been an issue however at this preliminary stage, the investigator's participation as teacher provided an intimate view of the feasibility of the sequence and the participants perspective that would not have been otherwise available. Computerassisted self-administered questionnaires have the advantage of eliciting more openness to sensitive information and are more accessible, however, to accommodate the needs of two participants in this special population, surveys were administered by phone which may have resulted in social desirability bias. ${ }^{75}$ Using mixed survey administration has been demonstrated to be effective in the visually impaired population, and indeed necessary in some cases, resulting in good data quality. 76

Future research should: i) use a run-in period with more than one visit prior to randomization to promote enrollment of participants who are likely to return for multiple study visits, ii) develop strategies to improve motivation, and iii) accommodate busy schedules with various class options to help increase and facilitate participation in the AYT. A future randomized controlled trial could determine whether the AYT is capable of producing a specific beneficial result (i.e. efficacy).

In the absence of a real placebo or sham alternative for yoga, one possibility is an active control group such as an education control. An education control could include didactic presentations corresponding to information about stress and anxiety, sleep disturbances, mindbody relationships, orientation and mobility, but without providing specific guidance on strategies to improve these areas. The purpose of the active control would be to provide participants with the equivalent attention from an instructor, matched for duration and frequency, social interactions with others and enjoyment of the protocol. As described in the above discussion, the next iteration of this study would benefit from mixed methods that would include more objective measures, as well as standard validated questionnaires.

Our recruitment efforts yielded a heterogeneous sample of participants. It may be important to distinguish between those with congenital blindness vs. those with acquired VI (e.g. RP) to determine whether there are differences either at baseline or in the responses to the AYT based on the duration of VI. Participants with congenital vision loss may be better adapted and therefore not as affected by balance problems (although see Stones and Kozma, 1987) ${ }^{77}$ or negative psychosocial states. With the exception of the balance and RR measures, the other outcomes for the psychosocial states were self-reported, and the 
subjects' responses may have been biased since it was not possible to mask the subjects to the intervention.

The positive responses during the exit surveys and the high participation rates among the majority of the sample help demonstrate the feasibility of the AYT. Participants also reported perceived benefits and improved quality of life. The 1 st cohort of four subjects had improved scores on the stress, anxiety and depression questionnaires, while the $2^{\text {nd }}$ cohort of three subjects had improved scores for the awareness, respiration, and balance measures. Sleep disturbances trended toward improvements in the 1st cohort, but to a lesser degree in the $2^{\text {nd }}$ cohort. These promising results warrant further investigation with a larger sample size and active control.

\section{References}

1. Congdon N, O'Colmain B, Klaver CCW, et al. Causes and prevalence of visual impairment among adults in the United States. Arch Ophthalmol 2004;122:477-85.

2. Dagnelie G. Visual prosthetics 2006: assessment and expectations. Expert Rev Med Devices 2006;3:315-25.

3. Shintani K, Shechtman DL, Gurwood AS. Review and update: current treatment trends for patients with retinitis pigmentosa. Optometry 2009;80:384-401.

4. Kiser AK, Pronovost PJ. Management of diseases without current treatment options something can be done. JAMA 2009;301:1708-9.

5. What is complementary and alternative medicine? Bethesda: National Center for Complementary and Alternative Medicine, National Institutes of Health; 2010. Available from: http://nccam.nih.gov/ health/whatiscam

6. Barnes PM, Bloom B, Nahin RL. Complementary and alternative medicine use among adults and children: United States, 2007. Natl Health Stat Report 2008:1-23.

7. Khalsa SBS. Yoga as a therapeutic intervention: a bibliometric analysis of published research studies. Indian J Physiol Pharmacol 2004;48:269-85.

8. Ospina MB, Bond K, Karkhaneh M, et al. Clinical trials of meditation practices in health care: characteristics and quality. J Altern Complement Med 2008;14:1199-213.

9. Kiser AK, Dagnelie G. Reported effects of non-traditional treatments and complementary and alternative medicine by retinitis pigmentosa patients. Clin Exp Optom 2008;91:166-76.

10. Gordo MA, Recio M, Sanchez-Barcelo EJ. Decreased sleep quality in patients suffer- ing from retinitis pigmentosa. J Sleep Res 2001;10:159-64.

11. Lockley SW, Skene DJ, Arendt J, et al. Relationship between melatonin rhythms and visual loss in the blind. J Clin Endocrinol Metabol 1997;82:3763-70.

12. Ionescu D, Driver HS, Heon E, et al. Sleep and daytime sleepiness in retinitis pigmentosa patients. J Sleep Res 2001;10: 329-35.

13. Provencio I, Rodriguez IR, Jiang GS, et al. A novel human opsin in the inner retina. $\mathrm{J}$ Neurosci 2000;20:600-5.

14. Altimus CM, Güler AD, Villa KL, et al. Rodscones and melanopsin detect light and dark to modulate sleep independent of image formation. Proc Natl Acad Sci 2008;105:19998-20003.

15. Manjunath NK, Telles S. Influence of Yoga and Ayurveda on self-rated sleep in a geriatric population. Indian J Med Res 2005; 121:683-90.

16. Khalsa SBS. Treatment of chronic insomnia with yoga: a preliminary study with sleepwake diaries. Appl Psychophysiol Biofeedback 2004;29:269-78.

17. Cohen L, Warneke C, Fouladi RT, et al. Psychological adjustment and sleep quality in a randomized trial of the effects of a Tibetan yoga intervention in patients with lymphoma. Cancer 2004;100:2253-60.

18. Massion AO, Teas J, Hebert JR, et al. Meditation, melatonin and breast/prostate cancer: hypothesis and preliminary data. Med Hypotheses 1995;44:39-46.

19. Harinath K, Malhotra AS, Pal K, et al. Effects of Hatha yoga and Omkar meditation on cardiorespiratory performance, psychologic profile, and melatonin secretion. Journal J Altern Complement Med 2004;10:261-8.

20. Tooley GA, Armstrong SM, Norman TR, Sali A. Acute increases in night-time plasma melatonin levels following a period of meditation. Biol Psychol 2000;53:69-78.

21. Folkard S, Arendt J, Aldhous M, Kennett H. Melatonin stabilizes sleep onset time in a blind man without entrainment of cortisol or temperature rhythms. Neurosci Lett 1990;113:193-8.

22. Heckenlively JR, Yoser SL, Friedman LH, Oversier JJ. Clinical findings and common symptoms in retinitis pigmentosa. Am J Ophthalmol 1988;105:504-11.

23. Bittner AK, Edwards L, George M. Coping strategies to manage stress related to vision loss and fluctuations in retinitis pigmentosa. Optometry 2010;81:461-8.

24. Berman K, Brodaty H. Psychosocial effects of age-related macular degeneration. Int Psychogeriatr 2006;18:415-28.

25. Chong CSM, Tsunaka M, Tsang HWH, et al. Effects of yoga on stress management in healthy adults: A systematic review.
Altern Ther Health Med 2011;17:32-8.

26. Pilkington K, Kirkwood G, Rampes H, Richardson J. Yoga for depression: the research evidence. J Affect Disord 2005;89: 13-24.

27. Kirkwood G, Rampes H, Tuffrey V, et al. Yoga for anxiety: a systematic review of the research evidence. Brit J Sport Med 2005;39:884-91;discussion 91.

28. Miller R. The psychophysiology of respiration: Eastern and Western perspectives. Int J Yoga Ther 1991;2:8-23.

29. Brown RP, Gerbarg PL. Sudarshan Kriya Yogic breathing in the treatment of stress, anxiety, and depression. Part II--clinical applications and guidelines. J Altern Complement Med 2005;11:711-7.

30. Grossman P. Respiration, stress, and cardiovascular function. Psychophysiology 1983;20:284-300.

31. Monnazzi P, Leri 0, Guizzardi L, et al. Antistress effect of yoga-type breathing: modification of salivary cortisol, heart rate and blood pressure following a stepclimbing exercise. Stress Health 2002;18:195-200.

32. Ray CT, Horvat M, Croce R, et al. The impact of vision loss on postural stability and balance strategies in individuals with profound vision loss. Gait Posture 2008; 28:58-61.

33. Geruschat DR, Turano KA, Stahl JW. Traditional measures of mobility performance and retinitis pigmentosa. Optometry 1998;75:525-37.

34. Felson DT, Anderson JJ, Hannan MT. Impaired vision and hip fracture. The Framingham Study. J Am Geriatr Soc 1989; 37:495-500.

35. Freeman EE, Muñoz B, Rubin G, West SK. Visual field loss increases the risk of falls in older adults: the Salisbury eye evaluation. Invest Ophthalmol Vis Sci. 2007; 48:4445-50.

36. Legood R, Scuffham P, Cryer C. Are we blind to injuries in the visually impaired? A review of the literature. Inj Prev 2002;8:155-60.

37. Wood JM, Lacherez P, Black AA, et al. Risk of Falls, injurious falls, and other injuries resulting from visual impairment among older adults with age-related macular degeneration. Invest Ophthalmol Vis Sci 2011;52:5088-92.

38. Gauchard G, Jeandel C, Tessier A, Perrin P. Beneficial effect of proprioceptive physical activities on balance control in elderly human subjects. Neurosci Lett 1999; 273:81-4.

39. Vellas B, Wayne S, Romero L, et al. One-leg balance is an important predictor of injurious falls in older persons. J Am Geriatr Soc 1997;45:735-8.

40. Mosston M. Developmental movement. Columbus, Ohio: Charles E. Merrill 
Publishing Company; 1965.

41. West CG, Gildengorin G, HaegerstromPortnoy G, et al. Is vision function related to physical functional ability in older adults? J Am Geriatr Soc 2002;50:136-45.

42. Hart CEF, Tracy BL. Yoga as steadiness training: effects on motor variability in young adults. J Strength Cond Res 2008;22:1659-69.

43. Tüzün S, Aktas I, Akarirmak U, et al. Yoga might be an alternative training for the quality of life and balance in postmenopausal osteoporosis. Eur J Phys Rehabil Med 2010;46:69-72.

44. Lavretsky H. Complementary and alternative medicine use for treatment and prevention of late-life mood and cognitive disorders. Aging Health 2009;5:61-78.

45. Shelov DV, Suchday S, Friedberg JP. A pilot study measuring the impact of yoga on the trait of mindfulness. Behav Cogn Psychother 2009;37:595-8.

46. Dunn K. The effectiveness of Hatha yoga on symptoms of anxiety and related vulnerabilities, mindfulness, and psychological wellbeing in female health care employees. 2009. Available from: http://digilib.gmu.edu:8080/dspace/bitstream/ 1920/5596/1/Dunn_Karen.pdf

47. Dersu I, Wiggins MN, Luther A, et al. Understanding Visual Fields, Part I; Goldmann Perimetry. Available from: http://centreforeyehealth.com.au/uploads/ 37864/ufiles/Dersu_et_al_2006_Goldman n_Visual_Fields.pdf

48. Ferris FL, Kassoff A, Bresnick GH, Bailey I. New visual-acuity charts for clinical research. Am J Ophthalmol 1982;94:91-6.

49. Pelli DG, Robson JG, Wilkins AJ. The design of a new letter chart for measuring contrast sensitivity. Clin Vis Sci 1988;2:187.

50. Kabat-Zinn J, Chapman-Waldrop A. Compliance with an outpatient stress reduction program: rates and predictors of program completion. J Behav Med 1988;11: 333-52.

51. Buysse DJ, Reynolds CF, Monk TH, et al. The Pittsburgh Sleep Quality Index: a new instrument for psychiatric practice and research. Psychiatry Res 1989;28:193-213.
52. Beck AT, Brown G, Epstein N, Steer RA. An inventory for measuring clinical anxiety psychometric properties. J Consult Clin Psychol 1988;56:893-7.

53. Beck AT, Erbaugh J, Ward CH, et al. An inventory for measuring depression. Arch Gen Psychiatry 1961;4:561-71.

54. Cohen S, Kamarck T, Mermelstein R. A global measure of perceived stress. J Health Soc Behav 1983;24:385-96.

55. Beck A, Steer RA, Garbin M. Psychometric properties of the beck depression inventory: twenty-five years of evaluation. Clin Psychol Rev 1988;8:77-100.

56. Leung DY, Lam T-h, Chan SS. Three versions of perceived stress scale: validation in a sample of Chinese cardiac patients who smoke. BMC Public Health 2010;10:513.

57. Benavides S, Caballero J. Ashtanga yoga for children and adolescents for weight management and psychological well being: an uncontrolled open pilot study. Complement Ther Clin Pract 2009;15:110-4.

58. Harner H, Hanlon AL, Garfinkel M. Effect of Iyengar Yoga on mental health of incarcerated women: a feasibility study. Nurs Res 2010;59:389-99.

59. Chen K-M, Chen M-H, Chao H-C, et al. Sleep quality, depression state, and health status of older adults after silver yoga exercises: cluster randomized trial. Int $\mathbf{J}$ Nurs Stud 2009;46:154-63.

60. Yim-Chiplis PK, Talbot LA. Defining and measuring balance in adults. Biol Res Nurs 2000;1:321-31.

61. Duncan P, Studenski S. Balance and gait measures. In: Lawton MP, Teresi JA. Annual Review of Gerontology and Geriatrics, vol 14. New York: Springer Publishing Company; 1994.

62. Briggs RC, Gossman MR, Birch R, et al. Balance performance among noninstitutionalized elderly women. Physical Therapy 1989;69:748-56.

63. Lim W, Carty S, Macfarlane J, et al. Respiratory rate measurement in adults how reliable is it? Resp Med 2002;96:31-3.

64. Tortora GJ, Anagnostakos NP. Principles of anatomy and physiology. 6th Edition. Harper \& Row: Wiley; 1990.
65. Sherwood L. Fundamentals of physiology: a human perspective. Thomson Brooks/ Cole; 2006.

66. Beckett BS. Illustrated Human and Social Biology. Oxford: Oxford University Press; 1995.

67. Cardaciotto L, Herbert JD, Forman EM, et al. The assessment of presentmoment awareness and acceptance: the Philadelphia mindfulness scale. Assessment 2008;15:204-23.

68. Bishop SR, Lau M, Shapiro S, et al. Mindfulness: a proposed operational definition. Clin Psychol Sci Pract 2004;11:23041.

69. Norman GR. Issues in the use of change scores in randomized trials. J Clin Epidemiol 1989;42:1097-105.

70. Carmody J, Crawford S, Churchill L. A pilot study of mindfulness-based stress reduction for hot flashes. Menopause 2006;13: 760-9.

71. Li F, Fisher KJ, Harmer P, et al. Tai chi and self-rated quality of sleep and daytime sleepiness in older adults: a randomized controlled trial. J Am Geriatr Soc 2004;52: 892-900.

72. King A, Oman R, Brassington G, et al. Moderate-intensity exercise and self-rated quality of sleep in older adults - a randomized controlled trial. JAMA 1997;277:32-7.

73. Woolery A, Myers H, Sternlieb B, Zeltzer L. A yoga intervention for young adults with elevated symptoms of depression. Altern Ther Health Med 2004;10:60-3.

74. Lee HKM, Scudds RJ. Comparison of balance in older people with and without visual impairment. Age and Ageing 2003;32:643-9.

75. Kreuter F, Presser S, Tourangeau R. Social desirability bias in CATI, IVR, and web surveys: the effects of mode and question sensitivity. Public Opin Q 2009;72:847-65.

76. De Leeuw E, Hox J, Kef S. Computerassisted self-interviewing tailored for special populations and topics. Field Methods 2003;15:223-51.

77. Stones M, Kozma A. Balance and age in the sighted and blind. Arch Phys Med Rehabil 1987;68:85. 